\title{
Vacuum Dependent Bell Local Hidden Variable Models and Generalized C.H.S.H. Inequalities
}

\author{
Gianpaolo Bei \\ Department of Basic and Applied Sciences for Engineering, Sapienza University of Rome, Rome, Italy \\ Email: gianpaolo.bei@uniroma1.it, capo.gian@libero.it
}

How to cite this paper: Bei, G. (2022) Vacuum Dependent Bell Local Hidden Variable Models and Generalized C.H.S.H. Inequalities. Journal of Applied Mathematics and Physics, 10, 11-20. https://doi.org/10.4236/jamp.2022.101002

Received: November 15, 2021

Accepted: January 4, 2022

Published: January 7, 2022

Copyright (c) 2022 by author(s) and Scientific Research Publishing Inc. This work is licensed under the Creative Commons Attribution International License (CC BY 4.0).

http://creativecommons.org/licenses/by/4.0/ (c) (i) Open Access

\begin{abstract}
We extend a previous model of the author which generalizes Bell local hidden variable models to the case of entangled photon pairs assuming that the standard Bell correlation functions depend on a hidden vacuum index. We deduce a generalization of Bell theorem assuming that classical observables are not dichotomic and that photon pair emission and detection is not a stationary stochastic process. We derive a photon imperfect polarization correlation functions due to rotational invariance breaking induced by hidden vacuum spin currents. We implement formally this approach deducing a generalization of C.H.S.H. inequalities which asymptotically converges to the standard one and which might be competitive with standard quantum mechanics predictions. We suggest to test this inequalities conceiving new E.P.R.-Bell like tests with time dependent detector efficiency and photon flux. Finally, we suggest to apply these generalized inequalities to the correlation functions of entangled classical spinning waves realized recently with metamaterials.
\end{abstract}

\section{Keywords}

Vacuum Index, Complex Hidden Variables, Vacuum Dependent Photons Imperfect Correlations, Vacuum Dependent Detector Efficiency, Generalized C.H.S.H. Inequalities

\section{Introduction}

Recently, a consistent group of researchers questioned the non contextual description of photon polarization used for deducing Bell theorem, arguing that Bell tests cannot be used to demonstrate intrinsic non local property of quantum states [1]-[6]. This new contextual classical reformulation of hidden variable theories gave strong support to the old research program of Einstein to build a deterministic theory which completes Quantum Mechanics. In fact, he struggled 
many years against Bohr to convince him that quantum wavefunctions were just temporary mathematical tools useful to describe emergent properties of ensemble of quantum particles, but not suitable to describe the dynamics of individual ones. Since its pioneer work, few researchers continued to look for a deterministic interpretation of quantum mechanics which was in opposition to the standard statistical one of Bohr, called the Copenhagen interpretation [7]. In particular, the debate concentrated on the realistic approach to quantum physics of beam of free particles produced by a common decaying source, as suggested in the famous paper of Einstein, Podolsky and Rosen [8]; they illustrated, in that work, a paradox of two separated quantum particle with opposite momentum aimed to show with a concrete example the incompleteness of standard of the Copenhagen interpretation and therefore invalidating, on their opinions, the foundations of quantum mechanics. This work inspired in the same year Schrodinger [9] to discover the entanglement properties of two coupled quantum systems. The subsequent debate on hidden variable models stimulated Bohm to develop his deterministic model as a counterexample to the famous no go theorem of Von Neumann.

This model, called then Bohmian Mechanics, was not conceived to context Quantum Mechanics stationary statistical predictions, but just to show that it was possible to build a classical model of point massive particles compatible with known experimental data, assuming the existence of electron space-time trajectories. Few years later [10], he reformulated and simplified E.P.R model substituting the impulse, used by Einstein and collaborators as hidden variable, with the spin of entangled electrons. Curiously during the same years, notwithstanding it emerged the role of vacuum fluctuations for explaining important phenomena such as the Lamb shift and the Casimir effect, the great majority of the quantum physics scientific community continued to adhere to the Copenhagen interpretation and was not interested in deterministic models of nuclear reactions and vacuum controlled quantum processes; its successful predictions concerned mostly expectation values derived using the statistical interpretation of Born and the controversial postulate of the instantaneous collapse of the wavefunction. As outlined in a recent paper of the author [11] since this dogmatic and contradictory point of view was applied successfully to nuclear physics, it was assumed to be the only possible framework to describe the experiment of high energy physics concerning asymptotic scattering of both stable and unstable particles.

We think that as some authors proposed recently [12] [13] that a realistic model of Quantum Physics must be based on deterministic models of vacuum fluctuations and its influence on the measurement process; over more since every quantum prediction depends on the vacuum fluctuations induced by the apparatus, we expect that it should abandon the postulate of instantaneous wavefunction collapse and should develop a deterministic non hamiltonian model of non instantaneous system-apparatus interaction. In particular, we suggest to conceive photon pair productions and detections as non stationary sto- 
chastic process that can be influenced from the environment and from dynamic vacuum fluctuations. We suggest to conceive the source photon emission as an unstable time dependent process, induced by deterministic vacuum fluctuations, that could be enhanced and controlled by external laser fields, and implemented by the time dependent energy bandwidth of the source (that will make the standard hidden variable complex valued [11]. We discuss in this paper a simple model which is aimed to show the possibility to violate with a classical deterministic model Bell like inequalities just implementing in the standard observables and correlation functions of photon polarization entangled states the dynamic effect of the vacuum index of refraction. We show that it is possible with this formalism to reproduce asymptotically the experimental predictions of quantum mechanics supposed to verify in Bell tests. Our proposal exploits an analogy between source induced photon pair creations and the unstable quantum states described in nuclear physics with the formalism called Gamow kets [14]; we critique the standard concept of photon beam as a perfect monochromatic electromagnetic wave, since it conflicts either with the Heisenberg energy-time uncertain relation and both with the finite time windows of every photon detectors.

We outline that from this contradictory framework originates the problem of photon pair identification and the consequent loophole, which, for some authors [15], invalidates Bell theorem.

We think that the standard interpretation of Bell tests might become less attractive if in the future will be possible to realize experiments with rotating detectors axis and Stern Gerlach like apparatus for light beams (or making time dependent polarizers Stokes parameters).

In the following paragraph we describe a particular simplified model of unstable photon pairs with vacuum dependent imperfect polarization correlations, whose polarization is not purely transversal, and we will deduce generalized inequalities that violate the standard Bell-C.H.S.H. ones. We suggest to compare this prediction with modern test on Bell theorem enhancing the fluctuations of photon emission with external laser, in a way similar to recent laser assisted nuclear reactions [16]. Finally, we will deduce a formula of vacuum dependent detector efficiency that we hope could be tested in future E.P.R. Bell like experiments with temperature controlled polarization axis and time dependent detector efficiencies.

\section{Model}

In this paragraph we illustrate our new model, inspired by a previous one of the author [11] which is based on an extension in the complex domain of Bell classical observables making them non dichotomic since they depend on the hidden vacuum index. Our approach exploits the concept of symmetry breaking photon vacuum fluctuations in analogy with some recent works which introduced the new idea of vacuum texture [13] and of vacuum spins currents [17]; we suggest to explain photon detector fluctuations as by-products of hidden light speed oscillations of electromagnetic waves with complex Poynting vector. Our alterna- 
tive approach is aimed to explain deterministically the anomalous photon correlations experimentally tested and conventionally interpreted as violations of Bell-C.H.S.H. inequalities due to property of entanglement and as intrinsic signature of the quantum nature of entangled photon pairs [18] [19]. The main idea of our proposal is, as explained in the introduction, that photon pair production in standard Bell tests is a non stationary process whose fluctuating photon flux does not satisfy at each instant classical conservation law (neither the energy, nor the momentum, nor the angular momentum of the decaying quantum source are perfectly constant). We believe, in fact, as recently suggested by some authors [20] [21] that nuclear and atomic decaying processes might be controlled by environment induced vacuum symmetry breaking effects. In particular we think that a hidden variable model of photon pair entanglement must abandon the standard view of the perfect monochromatic view of the photon $E=$ $h v$, and of static plane of polarization, assumptions on which are based modern theory of photon emission and detections (and are of course used, together with the Malus classical law, in modern interpretations of Bell tests). On the contrary we try to extend the recent proposal [11] of time dependent complex hidden variable theories to the supposed non stationary process of photon pair production, in a similar manner to those introduced by Gamow with its explanation of quantum tunnelling in nuclear reactions [14] (where the time dependent complex energy shift is due to the complex nature of the Poynting vector of the radiation emitted by the source).

We start by assuming that the vacuum dependent energy shift of the decaying source equals the energy bandwidth of each photon pair emitted by it

$$
\Delta E_{\text {Source }}=\Delta E_{\text {Photon }}=h \Delta v(n)
$$

with the first member given by the standard relation modified by the dependence on the vacuum index $n(2)$

$$
\Delta E_{\text {Source }}=\frac{h}{\Gamma(n)}
$$

where $\Gamma(n)$ is the vacuum dependent atomic lifetime. This hidden photon pair energy fluctuations can be interpreted as caused by vacuum instabilities which induces oscillations of the polarization plane (in a similar wave to what happen in optics with the phenomena of complex generalized Snell law). These photon flux oscillations can be implemented in our hidden variable model assuming the following generalization of standard Bell like local polarization variables [22] [23] (even if they could be used to describe electromagnetic intensities)

$$
A(a, \lambda, n)=A^{\prime}=[1+f(n)] A
$$

where $A$ 'is the non dichotomic variable of the first detector (an analogous relation is valid for the observable $B$ measured with the second detector and $f(n)$ is an unknown function to be determined; we note that in our framework, contrary to the Bell one, the averages values of these observables are not zero

$$
\left|\bar{A}^{\prime}\right| \neq 0
$$


(the complex values of these observable are caused, as explained, as the imperfect correlations, by the supposed hidden oscillation of the plane of polarization and vacuum dependent degree of polarization), where the average is defined as usual by the integral

$$
\bar{A}^{\prime}=\int A(a, \lambda, n) \rho(\lambda) \mathrm{d} \lambda
$$

with the constrain

$$
\lim _{n \rightarrow 1} A(a, \lambda, n)=A
$$

In our proposal, the photon polarization is therefore imperfect and the photon correlation functions must be generalized

$$
P^{\prime}(a, b, n)=\int A(a, \lambda, n) B(b, \lambda, n) \rho(\lambda) \mathrm{d} \lambda=P(a, b)\left(\frac{1+\bar{A}^{\prime} \bar{B}^{\prime}}{\sqrt{\sigma_{A^{\prime}}^{2} \sigma_{B^{\prime}}^{2}}}\right)
$$

where the numbers in the square root of the last equation member are the uncertainties of the respective fluctuating observables, $\rho$ is the Bell stationary probability distribution and the function $f(n)$ must satisfy the obvious condition

$$
\lim _{n \rightarrow 1} f(n)=0
$$

If we insert the relation (5) into the integral of Equation (7), we can deduce the vacuum dependent photon correlation function

$$
P^{\prime}(a, b, n)=[1+f(n)]^{2} P(a, b)
$$

We note that the function in the first member of this formula gives for parallel axis of the detectors an imperfect vacuum dependent photon correlation

$$
P^{\prime}(a, a, n)=[1+f(n)]^{2} P(a, a)=-[1+f(n)]^{2}
$$

It is simple to deduce from the relation (8), exploiting the imperfect anti-correlation

$$
A(a, a, n)[1+f(n)]=-B(a, a, n)
$$

the generalized Bell inequality [21]

$$
\left|P^{\prime}(a, b, n)-P^{\prime}(a, c, n)\right| \leq|1+f(n)|^{2}+P^{\prime}(b, c, n) .
$$

In a similar way we can substitute $P^{\prime}$ in the $S$ function, defined by [1]

$$
S=P(a, b)-P\left(a^{\prime}, b^{\prime}\right)+P\left(a^{\prime}, b\right)+P\left(a, b^{\prime}\right)
$$

which is the function experimentally tested measuring the joint photon counting rates in laboratories.

Inserting the new formula of the correlation function $P^{\prime}$ in the second member of (12), we can define the analogue $S^{\prime}$ function of our vacuum dependent hidden variable model

$$
\left|S_{H V}^{\prime}\right|=|1+f(n)|^{2} S
$$

We finally deduce the vacuum generalization of C.H.S.H. inequality [1] 


$$
S_{H V}^{\prime} \leq 2|1+f(n)|^{2}
$$

which, of course, converges, when the vacuum index goes to one, to the standard C.H.S.H. inequality [24].

This relation offers a new interpretation, compatible with the realistic approach of Einstein, and with the followers of the contextual approach to Bell tests [2] [3]; in fact it shows that new classical vacuum dependent hidden variable models can violate standard inequalities and are compatible with the empirical inequalities obtained by some authors [3] [25], taking in account the detector efficiency.

$$
S_{\text {Lab }} \leq \frac{4}{\eta}-2=4-2 \delta
$$

where $\eta$ is the detector efficiency and $\delta$ is an empirical number given by the ratio of the real number $N^{\prime}$ of photon coincidences divided by the total number $N$ of the photon generated by the source [3]

$$
\delta=\frac{N^{\prime}}{N} .
$$

This relation justifies, we think, the critiques of some authors to Bell test based on the detector efficiency loophole [15] and could be exploited to deduce an explicit formula of the vacuum index assuming

$$
\delta(n)=\varepsilon \frac{\Delta E(n)}{h\left(v_{1}+v_{2}\right)}
$$

where $\varepsilon$ is a phenomenological unknown number and denominator of the fraction is the energy of photon pair and the source energy bandwidth $\Delta E(n)$ can be found using a relation proposed in a recent model of the author on atomic spectra [26]

$$
\Delta E(n)=M c^{2}\left(\frac{1}{n^{2}}-1\right)
$$

with $M$ the source inertial mass and $c$ the light speed. Since we wish to have a model compatible with the experimentally tested inequality (15), we can deduce an explicit formula of the $f$ function introduced, making equal (15) to (16) and obtaining finally

$$
f(n)=\sqrt{\frac{2}{\eta}-1}-1=\sqrt{2-\delta}-1 .
$$

that gives the relation of on the vacuum inserting (18) and (19) in (20).

It is interesting to compare our prediction with that one of quantum mechanics, taking in account that the vectors used in realistic polarizers are not perfectly defined [3]. This systematic angle dispersion or error $\Delta \theta$ of the two beams emitted by the source could be implemented introducing complex vector axis in the photon correlation function predicted by Quantum Mechanics for the emitted singlet states [22] 


$$
P_{Q . M .}(\boldsymbol{a}, \boldsymbol{b})=-\boldsymbol{a} \cdot \boldsymbol{b}=-\cos (\theta)
$$

that will become

$$
P_{Q . M .}^{\prime}=-\cos \left(\theta^{\prime}\right)=-\cos (\theta+i \Delta \theta)=-\mathrm{e}^{-\Delta \theta(n)} \cos (\theta)
$$

where $\theta$ is the relative angle between the two detector axis.

Our generalized inequalities gives an alternative way to bypass Bell theorem, different from the followers of the contextual reformulation of quantum measurement and hidden variable models [2] [3], even if we agree with them that is unrealistic the Bell assumption of one singular probability distribution independent from the detectors settings (anyway we think it should be considered in these alternative approaches to make them time dependent).

We hope that this new model may stimulate new search of time dependent hidden variable models for realistic approach to unstable process of entangled pairs of decaying nuclear particles, excited atoms or ions (whose spin dependent nuclear forces are not well understood); we suggest that this non stationary stochastic processes, might be described by path dependent hidden variable models similar to our tentative one, and could be tested by modulating the detector efficiency with the laser induced temperature waves recently investigated $T(x, t, \omega)$ [27].

More generally, we believe that our framework could be adapted to describe new E.P.R. like experiments for verifying if they satisfy the proposed inequalities with variable experimental set up. We hope it could be possible in the near future to conceive new tests with classical entangled acoustic (or thermal waves), as those realized with acoustic metamaterials, which showed that acoustic vortices might have classical spin [28] [29] (it is natural to look at the matematerial acoustic index of refraction $n$ as the analogue of our vacuum index).

A classical wave entanglement model violating Bell like inequalities with continuous non dichotomic variables (as for example the wave intensities) will shed new light, we think, on the foundations of quantum statistics and the real meaning of the quantum wavefunctions (and maybe might be useful for the realization of quantum heat engines with time dependent violations of Carnot theorem).

Finally, we think it should be investigated more intensely vacuum induced space time symmetry breaking effects and memory effects for a realistic description of finite time process of entangled pair productions and annihilations and therefore for a non perturbative deterministic reformulation of Q.E.D which could deduce dynamically the fine structure constant in a similar way, maybe, to a semiclassical recent proposal [30]).

\section{Conclusions}

We illustrated in this paper that it is possible to conceive and implement a vacuum dependent local hidden variable model of photon imperfect polarization entanglement that violates standard Bell inequalities and that generalizes C.H.S.H. inequalities. We gave an interpretation of Bell test, therefore, opposite to the stan- 
dard ones. We introduced a vacuum dependent photon pair flux which makes the Bell conventional correlation functions dependent on time. We derived Bell generalized inequalities assuming a vacuum dependent detector efficiency. We suggested to test this generalized inequalities conceiving new experiments with laser induced time dependent photon emission and introducing a hidden temperature dependent oscillation in the detector efficiency of the photon polarizers. We proposed to apply this generalized hidden variable formalism to describe entanglement of acoustic vortex waves generated in modern meta materials.

Finally, we discussed how to extend this deterministic approach to general unstable processes concerning entangled pairs usually observed in nuclear fragment dynamics and in atomic excitation processes. We hope that this proposal will pave the way to the search of new non-perturbative deterministic reformulations of Q.E.D theory and of vacuum polarization and will offer new insights for future more accurate deterministic models of scattering processes of ions and high energy unstable particles.

\section{Conflicts of Interest}

The author declares no conflicts of interest regarding the publication of this paper.

\section{References}

[1] Hess, K. (2021) What Do Bell-Tests Prove? A Detailed Critique of Clauser-HorneShimony-Holt Including Counterexamples. Journal of Modern Physics, 12, 1219-1236. https://doi.org/10.4236/jmp.2021.129075

[2] Sica, L. (2020) The Bell Inequalities: Identifying What Is Testable and What Is Not. Journal of Modern Physics, 11, 725-740. https://doi.org/10.4236/jmp.2020.115047

[3] Kupczynski, M. (2020) Is the Moon There If Nobody Looks: Bell Inequalities and Physical Reality. Frontiers in Physics, 8, Article No. 273. https://doi.org/10.3389/fphy.2020.00273

[4] Jung, K. (2020) Polarization Correlation of Entangled Photons Derived without Using Non-Local Interactions. Frontiers in Physics, 8, Article No. 170. https://doi.org/10.3389/fphy.2020.00170

[5] Santos, E. (2020) Local Realistic Interpretation of Entangled Photon Pairs in the Weyl-Wigner Formalism. Frontiers in Physics, 8, Article No. 191. https://doi.org/10.3389/fphy.2020.00191

[6] Bohm, D. (1952) A Suggested Interpretation of the Quantum in Terms of Hidden Variables I. Physical Review, 85, 166-179. https://doi.org/10.1103/PhysRev.85.166

[7] Von Neumann, J. (1932) Mathematical Foundations of Quanatum Mechanics. Princeton University Press, Princeton.

[8] Einstein, A., Podolsky, A. and Rosen, N. (1935) Can Quantum Mechanical Description of Physical Reality Be Considered Complete? Physical Review, 47, 770-780. https://doi.org/10.1103/PhysRev.47.777

[9] Schrödinger, E. (1935) Die gegenwärtige Situation in der Quantenmechanik. Naturwissenshaften, 23, 807-812. https://doi.org/10.1007/BF01491891

[10] Bohm, D. and Aharonov, Y. (1957) Discussion of Experimental Proof for the Para- 
dox of Einstein, Rosen, and Podolsky. Physical Review, 108, 1070-1076. https://doi.org/10.1103/PhysRev.108.1070

[11] Bei, G. (2021) A Path Integral Generalization of Bell Local Hidden Variable Models for Unstable Particles. Journal of Applied Mathematics and Physics, 9, 2430-2438. https://doi.org/10.4236/jamp.2021.910154

[12] t'Hooft, G. (2021) Fast Vacuum Fluctuations and the Emergence of Quantum Mechanics. Foundation of Physics, 51, Article No. 63. https://doi.org/10.1007/s10701-021-00464-7

[13] Suzuki, Y. and Mertes, K.M. (2019) Vacuum Texture: A New Interpretation of Quantum Mechanics and a New Loophole for Bell's Inequality Measurements That Preserves Local Realism and Casuality.

[14] Gamow, G. (1928) Zur Quantentheorie des Atomkernes. Physikalische Zeitschrift, 51, 204-212. https://doi.org/10.1007/BF01343196

[15] De Raedt, H., Michielsen, K. and Hess, K. (2017) The Photon Identification Loophole in EPRB Experiments: Computer Models with Single-Wing Selection. Open Physics, 15, 713-733. https://doi.org/10.1515/phys-2017-0085

[16] Queisser, F. and Schützhold, R. (2019) Dynamically Assisted Nuclear Fusion. Physical Review C, 100, 041601(R). https://doi.org/10.1103/PhysRevC.100.041601

[17] Chu, C. and Leung, C. (2021) Induced Quantized Spin Current in Vacuum. Physical Review Letters, 127, Article ID: 111601.

https://doi.org/10.1103/PhysRevLett.127.111601

[18] Aspect, A., Grangier, P. and Roger, G. (1981) Experimental Tests of Realistic Local Theories via Bell's Theorem. Physical Review Letters, 47, 460-463. https://doi.org/10.1103/PhysRevLett.47.460

[19] Aspect, A., Dalibard, J. and Roger, G. (1982) Experimental Test of Bell's Inequalities Using Time-Varying Analyzers. Physical Review Letters, 49, 1804-1807. https://doi.org/10.1103/PhysRevLett.49.1804

[20] Bei, G. (2021) A Hidden Variable Model of Nuclear Reactions. International Journal of Applied Pharmaceutics, 8, 1-4. https://doi.org/10.14445/23500301/IJAP-V8I2P101

[21] Fischbach, E., Buncher, J.B., Gruenwald, J.T., Jenkins, J.H., Krause, D.E., Mattes, J.J., et al. (2009) Time-Dependent Nuclear Decay Parameters: New Evidence for New Forces? Space Science Reviews, 145, 285-335. https://doi.org/10.1007/s11214-009-9518-5

[22] Bell, J.S. (1964) On the Einstein-Podolsky-Rosen Paradox. Physics, 1, 195-200. https://doi.org/10.1103/PhysicsPhysiqueFizika.1.195

[23] Chen, G.Y. (2019) Collapse of Bell's Theorem. Journal of Modern Physics, 10, 1157-1165. https://doi.org/10.4236/jmp.2019.1010076

[24] Clauser, J.F., Horne, M.A., Shimony, A. and Holt, R.A. (1969) Proposed Experiment to Test Local Hidden Variable Theories. Physical Review Letters, 23, 880-884. https://doi.org/10.1103/PhysRevLett.23.880

[25] Nordén, B. (2016) Quantum Entanglement: Facts and Fiction-How Wrong Was Einstein after All? Quarterly Reviews of Biophysics, 49, e17. https://doi.org/10.1017/S0033583516000111

[26] Bei, G. (2021) A Hidden Variable Model of Hydrogen Energy Spectra. International Journal of Applied Pharmaceutics, 8, 44-46. https://doi.org/10.14445/23500301/IJAP-V8I1P107

[27] Li Voti, R. and Bertolotti, M. (2021) Thermal Waves Emitted by Moving Sources 
and the Doppler Effect. International Journal of Heat and Mass Transfer, 176, Article ID: 121098. https://doi.org/10.1016/j.ijheatmasstransfer.2021.121098

[28] Bliokh, K.Y. (2021) Spatiotemporal Vortex Pulses: Angular Momenta and SpinOrbit Interaction. Physical Review Letters, 126, Article ID: 243601. https://doi.org/10.1103/PhysRevLett.126.243601

[29] Bliokh, K. and Nori, F. (2019) Transverse Spin and Surface Waves in Acoustic Meta-Materials. Physical Review B, 99, 020301(R). https://doi.org/10.1103/PhysRevB.99.020301

[30] Alippi, A. (2020) Evaluation of the Fine Structure Constant. Journal of Modern Physics, 11, 1918-1925. https://doi.org/10.4236/jmp.2020.1112120 